\title{
Borel Summability in the Disorder Parameter of the Averaged Green's Function for Gaussian Disorder *
}

\section{F. Constantinescu, K. Klöckner, and U. Scharffenberger}

Institut für Angewandte Mathematik, Universität Frankfurt am Main, D-6000 Frankfurt, Federal Republic of Germany

\begin{abstract}
In this note we prove Borel summability in the disorder parameter of the averaged Green's function $\langle G(E, x, y)\rangle_{\gamma}$ of tight binding models

with Gaussian disorder

$$
H_{V}=-\Delta+V
$$

$$
d \lambda(V)=(2 \pi \gamma)^{-1 / 2} \exp \left(-V^{2} / 2 \gamma\right) d V
$$

for $\gamma \rightarrow 0$ and fixed large $|E|$. Using this, we can reconstruct the density of states $\varrho(E)_{\gamma}$ from the Borel sums of $\langle G(E, x, x)\rangle_{\gamma}$ with $\operatorname{Im} E \nearrow 0$ and $\operatorname{Im} E \searrow 0$.
\end{abstract}

\section{Introduction}

The motion of a quantum mechanical particle in a random potential on the lattice $Z^{v}$ is described by Anderson's tight binding model with dynamics governed by a random Hamiltonian

$$
H_{V}=-\Delta+V \text {. }
$$

Here $\Delta$ is the lattice Laplace operator and $V$ is a random potential belonging to the probability space

$$
\Omega=\prod_{j \in Z^{v}}\left(\mathbb{R}, d \lambda\left(V_{j}\right)\right),
$$

where $V_{j}$ is the value of $V$ at $j$ and the distribution of the $V_{j}$ is Gaussian and independent of $j$. We set

$$
d \lambda(V)=(2 \pi \gamma)^{-1 / 2} \exp \left(-V^{2} / 2 \gamma\right) d V
$$

where $\gamma$ is the disorder parameter.

The model specified by (1.1)-(1.3) is also called a disordered system with diagonal Gaussian disorder. Spectral properties of $H_{V}$ have been analyzed by Kunz and Souillard [9].

* Supported in part by the Deutsche Forschungsgemeinschaft 
The above model can be mapped onto the $n \rightarrow 0$ limit of a $\lambda \varphi^{4}$ quantum field theory continued analytically in the mass and the coupling constant. The energy $E$ of the disordered system then corresponds to the negative (!) mass $-m^{2}$ and the disorder parameter $\gamma$ corresponds to $-g^{-1}$. For a detailed study of this relationship see Constantinescu, Fröhlich and Spencer [2].

For $|\gamma| \gg 1$ or $|E| \gg 1$ we have a convergent random path expansion $[2,5]$ for the averaged Green's function which will be the starting point of our discussion. We closely follow the notation developped in [2]. The expansion is of the form

$$
\langle G(E, x, y)\rangle=\sum_{\omega: x \rightarrow y} \prod_{j \in \omega} \int(2 v-E+V)^{-n_{j}(\omega)} d \lambda(V),
$$

where $\omega$ is a random path and $n_{j}(\omega)$ is the number of times $\omega$ visits $j \in Z^{v}$.

One can also try to expand the averaged Green's function in a more traditional perturbation series

$$
\langle G(E, x, y)\rangle=\sum_{N=0}^{\infty} \sum_{\left\{x_{1}, \ldots, x_{N}\right\}} \prod_{j=0}^{N} G_{0}\left(E, x_{j}, x_{j+1}\right)\left\langle\prod_{j=1}^{N} V_{x_{j}}\right\rangle,
$$

where $G_{0}$ is the free Green's function, $x_{0}=x, x_{N+1}=y$ and $\langle>$ denotes averaging with respect to $d \lambda(V)$. In [13] Wegner makes use of this formal perturbation expansion in order to derive an exact formula for the density of states of a model describing the quantized Hall-effect. It turns out that the expansion for the Green's function of this model coincides with the expansion for the two-point function of a zero-dimensional complex $\left(\phi^{*} \phi\right)^{2}$ model. In an addendum Wegner is able to show factorial bounds for the moments of the density of states which is sufficient to ensure that the function is uniquely determined by perturbation expansion. Stimulated by this work we prove Borel summability properties of the expansion (1.4) in the disorder parameter $\gamma$ for the averaged Green's function in the tight binding model with Gaussian disorder. Unfortunately we cannot control $\langle G(E, x, y)\rangle$ for all energies $E$, so we must restrict ourselves to $|E|$ large.

Our arguments also yield a reconstruction of the density of states $\varrho$ from the perturbation series of $\langle G(E, x, x)\rangle_{\gamma} \cdot \varrho$ is not Borel summable, in fact Constantinescu-Fröhlich-Spencer [2] prove exponential decay as $\gamma \rightarrow 0$. Properties of $\langle G\rangle$ are derived from those of the one-dimensional integrals by Vitali's theorem and combinatorial estimates for terms contributing to the $k^{\text {th }}$ order in $\gamma$ of the random path expansion.

\section{The Random Path Expansion.}

\section{Analyticity of the Averaged Green's Function}

We will start by analyzing the random path expansion (1.4) and prove its convergence for fixed large $|E|$ and $|\gamma|$ small independent of $|E|$. We rewrite (1.4) as

with

$$
\langle G(E, x, y)\rangle_{\gamma}=\sum_{\omega: x \rightarrow y} \prod_{j \in \omega} I_{n_{j}}(\gamma, E)
$$

$$
I_{r}(\gamma, E)=(2 \pi \gamma)^{-1 / 2} \int_{-\infty}^{\infty} \exp \left(-V^{2} / 2 \gamma\right)(2 v-E+V)^{-r} d V
$$

for $r \geqq 1$. 
Our proof is organized as follows: We first show that the random path expansion is convergent for complex $\gamma$ and $E$ with $|E|$ large and $|\gamma|$ small which yields the required analytic continuation of $\langle G(E, x, y)\rangle_{\gamma}$ to a sector with opening angle greater than $\pi$ with the real axis in its interior. This is not quite sufficient for "ordinary" Borel summability, but we can show that the function with rotated argument is Borel summable in a region which again includes an interval $\left(0, R^{\prime}\right)$, $R^{\prime}>0$, on the real axis. We call this "generalized" Borel summability.

The relations (2.1) and (2.2) are defined for $\operatorname{Im} E \neq 0$. In what follows we shall consider two different functions $I_{r}^{+}$(respectively $I_{r}^{-}$) which are defined by (2.2) restricted to the upper (respectively lower) halfplane and similarly two different branches $\left\langle G^{+}(E, x, y)\right\rangle$ and $\left\langle G^{-}(E, x, y)\right\rangle$ of the Green's function are defined by (2.1). We use the formula

$$
(2 v-E+V)^{-r}=\frac{i^{r}}{(r-1) !} \int_{0}^{\infty} t^{r-1} \exp (-i t(2 v-E+V)) d t
$$

valid for $\operatorname{Im} E>0$ in order to study $I_{r}^{+}$. Inserting (2.3) into (2.2), applying Fubini's theorem and making use of the Fourier transform of $\exp \left(-V^{2} / 2 \gamma\right)$ we obtain:

$$
I_{r}^{+}(\gamma, E)=\frac{i^{r}}{(r-1) !} \int_{0}^{\infty} t^{r-1} \exp (-i t(2 v-E)) \exp \left(-\gamma t^{2} / 2\right) d t .
$$

This integral converges for arbitrary $E$ and $\gamma$ with $\operatorname{Re} \gamma>0$ and can be regarded as an analytic continuation of (2.2) beyond the real $E$-axis from above.

Using a similar procedure for $\operatorname{Im} E<0$ we obtain:

$$
I_{r}^{-}(\gamma, E)=\frac{(-i)^{r}}{(r-1) !} \int_{0}^{\infty} t^{r-1} \exp (+i t(2 v-E)) \exp \left(-\gamma t^{2} / 2\right) d t,
$$

which for real $E$ is exactly the complex conjugate of (2.4). $I_{r}^{+}$and $I_{r}^{-}$are studied in an appendix. We recall some properties of $I_{r}^{ \pm}$in order to derive regularity properties of $\left\langle G^{+}\right\rangle$and $\left\langle G^{-}\right\rangle$.

Let $\theta=\arg (2 v-E)$, and define

$$
\begin{aligned}
& S_{\theta, \varepsilon}^{+}=\{\gamma:-5 \pi / 2+2 \theta+\varepsilon<\arg \gamma<\pi / 2+2 \theta-\varepsilon\}, \\
& S_{\theta, \varepsilon}^{-}=\{\gamma:-\pi / 2+2 \theta+\varepsilon<\arg \gamma<5 \pi / 2+2 \theta-\varepsilon\} .
\end{aligned}
$$

By (A.1) and (A.2) $I_{r}^{+}$and $I_{r}^{-}$are analytic in $\gamma$ in $S_{\theta, \varepsilon}^{+}$and $S_{\theta, \varepsilon}^{-}$respectively. Furthermore by (A.13) we have the bound

$$
\left|I_{r}^{ \pm}(\gamma, E)\right| \leqq \frac{C_{2}(\varepsilon,|\gamma|)^{r}}{|2 v-E|^{r}},
$$

for $\gamma \in S_{\theta, \varepsilon}^{+}$and $S_{\theta, \varepsilon}^{-}$respectively. Here $C_{2}(\varepsilon,|\gamma|) \rightarrow \infty$ for $\varepsilon \rightarrow 0$.

Now if $|2 v-E|$ is large enough and $\gamma \in S_{\theta, \varepsilon}^{ \pm}$, we can sum the expansion (2.1) absolutely, majorizing it by the expansion for a free Green's function with positive mass $m$ (cf. Constantinescu et al. [2]).

Using Vitali's theorem we obtain:

Theorem 1. For every $\varepsilon>0$ there exist constants $C_{\varepsilon}>0$ and $E_{\varepsilon}>0$ such that $\left\langle G^{ \pm}(E, x, y)\right\rangle_{\gamma}$ are analytic functions in the regions:

$$
\left\{E:|2 v-E|>E_{\varepsilon}\right\} \times\left\{\gamma: y \in S_{\theta, \varepsilon}^{ \pm},|\gamma|<C_{\varepsilon}\right\},
$$

where $S_{\theta, \varepsilon}^{ \pm}$are described by (2.5) and (2.5') respectively. 


\section{Asymptoticity of the $\boldsymbol{\gamma}$-Expansion for the Averaged Green's Function}

Now we derive the expansion for $\left\langle G^{ \pm}(E, x, y)\right\rangle_{\gamma}$ by summing over all random paths $\omega$ from $x$ to $y$. We insert (A.9) into (2.1) and use

$$
\prod_{i=1}^{N} \sum_{j=0}^{M} a_{i j}=\sum_{j_{1}, \ldots, j_{N}=0}^{M} \prod_{i=1}^{N} a_{i j_{i}} \text {. }
$$

Terminating the expansion of the $I_{r}(\gamma, E)$ at the $M^{\text {th }}$ order, we obtain

$$
\begin{aligned}
\left\langle G^{ \pm}(E, x, y)\right\rangle_{\gamma}= & \sum_{\omega: x \rightarrow y} \pi^{-|\omega| / 2} \sum_{j_{1}, \ldots, j_{|\omega|}=1}^{M} \prod_{i=1}^{|\omega|} \Gamma\left(j_{i}+1 / 2\right)\left(\begin{array}{c}
n_{i}+2 j_{i}-1 \\
n_{i}-1
\end{array}\right) \\
& \cdot(2 \gamma)^{j_{i}}(2 v-E)^{-n_{i}-2 j_{i}}
\end{aligned}
$$

+ sum over products involving remainder terms.

$|\omega|$ is the number of points visited by $\omega$ (without counting multiplicities). Thus the sum over all terms of order $k, k \leqq M$, is

$$
\begin{aligned}
& a_{k}(\gamma)=(2 \gamma)^{k} \sum_{\omega: x \rightarrow y} \pi^{-|\omega| / 2} \prod_{j=1}^{|\omega|}(2 v-E)^{-n_{j}} \\
& \sum_{j_{1}+\ldots+j_{|\omega|}=k} \prod_{i=1}^{|\omega|} \Gamma\left(j_{i}+1 / 2\right)\left(\begin{array}{c}
n_{i}+2 j_{i}-1 \\
n_{i}-1
\end{array}\right)(2 v-E)^{-2 j_{i}} .
\end{aligned}
$$

We have to show that (3.3) is absolutely convergent for $|2 v-E|$ large. Note that the binomial coefficients are the weights of the negative binomial distribution (Feller [4]). We have for $0 \leqq p \leqq 1$,

$$
\sum_{j=0}^{\infty}\left(\begin{array}{c}
r+j-1 \\
r-1
\end{array}\right) p^{j}(1-p)^{r}=1
$$

First we estimate the inner sum:

$$
\begin{aligned}
& \quad \sum_{j_{1}+\ldots+j_{|\omega|}=k} \prod_{i=1}^{|\omega|} \Gamma\left(j_{i}+1 / 2\right)\left(\begin{array}{c}
n_{i}+2 j_{i}-1 \\
n_{i}-1
\end{array}\right)|2 v-E|^{-2 j_{i}} \\
& \quad \leqq k ! \sum_{j_{1}+\ldots+j_{|\omega|}=k} \prod_{i=1}^{|\omega|}\left(\begin{array}{c}
n_{i}+2 j_{i}-1 \\
n_{i}-1
\end{array}\right)|2 v-E|^{-2 j_{i}} \\
& \leqq k ! \sum_{j_{1}, \ldots, j_{|\omega|}=0}^{\infty} \prod_{i=1}^{|\omega|}\left(\begin{array}{c}
n_{i}+2 j_{i}-1 \\
n_{i}-1
\end{array}\right)|2 v-E|^{-2 j_{i}} \\
& =k ! \prod_{i=1}^{|\omega|} \sum_{j=0}^{\infty}\left(\begin{array}{c}
n_{i}+2 j-1 \\
n_{i}-1
\end{array}\right)|2 v-E|^{-2 j} \\
& \leqq k ! \prod_{i=1}^{|\omega|}\left(\frac{|2 v-E|}{|2 v-E|-1}\right)^{n_{i}} \times \prod_{i=1}^{|\omega|}(2 v-E)^{-n_{i}}
\end{aligned}
$$

The sum over these products converges if $|2 v-E|-1>2 v$ and the term of order $k$ is bounded by

$$
\left|a_{k}(\gamma)\right| \leqq|2 \gamma|^{k} k ! \sum_{\omega: x \rightarrow y} \pi^{-|\omega| / 2}(|2 v-E|-1)^{-\sum_{i \in \omega} n_{i}(\omega)}
$$


It remains to estimate the error term. First we consider the remainder terms of the products $\prod_{i=1}^{|\omega|} I_{n_{i}}^{ \pm}(\gamma, E)$. We use the following

Lemma (Auberson-Mennessier [1]). Let

$$
\begin{aligned}
& f(z)=\sum_{n=0}^{M} f_{n} z^{n}+R_{M+1}^{f}(z), \\
& g(z)=\sum_{n=0}^{M} g_{n} z^{n}+R_{M+1}^{g}(z),
\end{aligned}
$$

with $\left|R_{M+1}^{f}\right| \leqq A^{f}(M+1) ! \sigma^{M+1}|z|^{M+1},\left|R_{M+1}^{g}\right| \leqq A^{g}(M+1) ! \sigma^{M+1}|z|^{M+1}$. Then the following expansion holds for $h(z)=f(z) g(z)$ :

$$
h(z)=\sum_{n=0}^{M} h_{n} z^{n}+R_{M+1}^{h}(z),
$$

with $\left|R_{M+1}^{h}(z)\right| \leqq 3 A^{f} A^{g}(M+1) ! \sigma^{M+1}|z|^{M+1}$.

This can easily be generalized to products $h=\prod_{i=1}^{n} f_{i}$. Here

$$
\left|R_{M+1}^{h}(z)\right| \leqq 3^{n-1} \prod_{i=1}^{n} A^{f_{i}}(M+1) ! \sigma^{M+1}|z|^{M+1}
$$

Let $\tilde{R}_{N+1}^{\omega, \pm}(\gamma, E)$ be the error term for the product $\prod_{i=1}^{|\omega|} I_{n_{j}}^{ \pm}(\gamma, E)$. Then we have, using (A.9) and (3.6):

$$
\left|\tilde{R}_{N+1}^{\omega, \pm}(\gamma, E)\right| \leqq(N+1) ! \frac{C_{2}^{N+1}|\gamma|^{N+1}}{|2 v-E|^{2(N+1)}} \prod_{i=1}^{|\omega|} \frac{\left(3 C_{1}\right)^{n_{i}}}{|2 v-E|^{n_{i}}}
$$

where $C_{1}, C_{2}$ are independent of $N$. If $|2 v-E|$ is large enough, we can sum over $\omega$ and obtain

$$
\left|R_{N+1}^{\langle G\rangle \pm}(\gamma, E)\right| \leqq(N+1) ! C_{3}\left(\frac{C_{2}}{|2 v-E|^{2}}\right)^{N+1}|\gamma|^{N+1} .
$$

We summarize the results in

Theorem 2. For every $N>0$ and $|2 v-E|$ large enough (independent of $N$ ), the averaged Green's function has the asymptotic expansion

$$
\left\langle G^{ \pm}(E, x, y)\right\rangle_{\gamma}=\sum_{k=0}^{N} a_{k}(\gamma)+R_{N+1}^{\langle G\rangle}{ }^{ \pm}(\gamma, E)
$$

with $k^{\text {th }}$ order terms given by (3.3) and $R_{N+1}^{\langle G\rangle}{ }^{ \pm}$obeying a factorial bound (3.8). The expansion is valid in $S_{\theta, \varepsilon}^{ \pm}$.

\section{Discussion of the Main Result}

Combining Theorems 1 and 2 we now obtain Borel summability in the following generalized sense (see Fig. 1). 


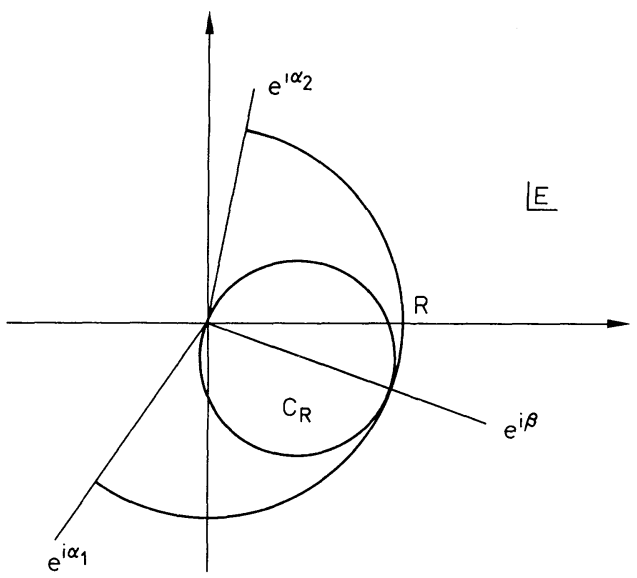

$C_{R}=$ Domain of validity of $(4.3)$

Let $f$ be analytic in a sector $S_{\alpha_{1}, \alpha_{2}}=\left\{z: \alpha_{1}<\arg z<\alpha_{2}, 0<|z|<R\right\}$ with $\alpha_{2}-\alpha_{1}=\pi+2 \varepsilon$. Let $f$ have an asymptotic expansion

$$
f(z) \sim \sum_{n=0}^{\infty} f_{n} z^{n}
$$

for $|z| \rightarrow 0$ in $S_{\alpha_{1}, \alpha_{2}}$ with factorial bounds

$$
\left|f(z)-\sum_{n=0}^{N} f_{n} z^{n}\right| \leqq A \sigma^{N+1}(N+1) !|z|^{N+1} .
$$

Then $f_{\beta}(z)=f\left(z e^{i \beta}\right)$ with $\beta=\frac{\alpha_{1}+\alpha_{2}}{2}$ is analytic in $S=\left\{z,-\frac{\pi}{2}-\varepsilon<\arg z<\frac{\pi}{2}+\varepsilon\right.$, $0<|z|<R\}$ and there is an asymptotic expansion

with

$$
f_{\beta}(z) \sim \sum_{n=0}^{N} f_{n}^{\beta} z^{n}
$$

$$
\left|f_{\beta}(z)-\sum_{n=0}^{N} f_{n}^{\beta} z^{n}\right| \leqq A \sigma^{N+1}(N+1) !|z|^{N+1} \text {, }
$$

where

$$
f_{n}^{\beta}=f_{n} e^{i \beta n}
$$

By Watson's theorem [12] we know that $f_{\beta}$ is Borel summable, i.e.

I) $B_{\beta}(t)=\sum \frac{f_{n}^{\beta}}{n !} t^{n}$ converges for $|t|<\sigma^{-1}$

II) $B_{\beta}(t)$ can be continued analytically to $|\arg t|<\varepsilon$ and satisfies

(*) $\left|B_{\beta}(t)\right|=O(\exp (t / R))$ for $t \rightarrow \infty$

III) $f_{\beta}(z)=z^{-1} \int_{0}^{\infty} \exp (-t / z) B_{\beta}(t) d t$ for $\operatorname{Re} z^{-1}>R^{-1}$. 
This implies

$$
f(z)=e^{i \beta} z^{-1} \int_{0}^{\infty} \exp \left(-t e^{-i \beta} / z\right) B_{\beta}(t) d t \text { for } \operatorname{Re}\left(z^{-1} e^{i \beta}\right)>R^{-1} .
$$

This includes a segment $(0, R \cos \beta)$ of the real axis, if $\beta$ has been chosen with $|\beta|<\frac{\pi}{2}$ (i.e. if $\left.(0, R) \subset S_{\alpha_{1}, \alpha_{2}}\right)$ (see Fig. 1). Thus $f(z)$ can be uniquely recovered from the Borel transform of $f_{\beta}$ for $z$ real which is often the physically interesting case.

This notion of "generalized Borel summability" can also be applied to Nevanlinna-Sokal's version of Watson's theorem $[10,12]$. In our case we have:

Theorem 3. For $|E|$ large enough the two branches of the averaged Green's function $\left\langle G^{ \pm}(E, x, y)\right\rangle_{\gamma}$ are Borel summable in a generalized sense for $|\gamma| \rightarrow 0$ in domains including a segment of the real axis, i.e. for $\arg (2 v-E)$ small there exist $\beta^{+}$and $\beta^{-}$, $\left|\beta^{ \pm}\right|<\pi / 2$, such that $\left\langle G^{ \pm}(E, x, y)\right\rangle\left(\gamma e^{i \beta \pm}\right)$ is Borel summable (in the usual sense).

Remark. The density of states $\varrho$ defined by

$$
\begin{aligned}
\varrho(E)_{\gamma} & =\lim _{\delta \rightarrow 0} \frac{1}{2 i}\{\langle G(E+i \delta, x, x)\rangle-\langle G(E-i \delta, x, x)\rangle\} \\
& =\frac{1}{2 i}\left\{\left\langle G^{+}(E, x, x)\right\rangle_{\gamma}-\left\langle G^{-}(E, x, x)\right\rangle_{\gamma}\right\}
\end{aligned}
$$

is not Borel summable for $|\gamma| \rightarrow 0$.

Since the bounds in Sects. 2 and 3 are independent of $\delta$ we can use Vitali's theorem to obtain analyticity of $\varrho(E)_{\gamma}$ as a function of $\gamma$ in the domain $S_{\theta=0, \varepsilon}^{+}$ $\cap S_{\theta=0, \varepsilon}^{-}$. However, the opening angle of this domain is $\pi-2 \varepsilon$, which is not enough for Borel summability. Indeed, if we try to insert (3.2) into (4.4) we find that the expansion for $\varrho$ is zero in every order. Nevertheless $\varrho$ can be reconstructed as in (4.4) once we know $\left\langle G^{+}\right\rangle$and $\left\langle G^{-}\right\rangle$on the real $E$-axis.

Our methods can also be applied to other disorder distributions, although not all results will remain valid. In particular, the summability techniques of Chap. 3 may be of interest for other distributions. One can easily check that given appropriate analyticity properties for a distribution

$$
d \lambda(V)=\gamma^{1 / 2} w\left(\gamma^{-1} V\right) d V,
$$

with $\int w(V) d V=1$, bounds of the form $\int V^{2 n} w\left(V^{2}\right) d V \leqq C A^{n} n$ ! are sufficient for factorial bounds on the remainder terms of an asymptotic expansion for $\langle G\rangle$.

\section{Appendix}

In this appendix we want to study the 1-dimensional integrals $I_{r}^{+}$and $I_{r}^{-}$. We rotate the contour of integration by the angle $\alpha=-(\arg \gamma) / 2$ and scale the integration variable by $1 / \sqrt{|\gamma|}$. Then by Cauchy's theorem it is easy to see that $I_{r}^{+}$ and $I_{r}^{-}$become:

$$
\begin{gathered}
I_{r}^{+}(\gamma, E)=\frac{i^{r} \gamma^{-r / 2}}{(r-1) !} \int_{0}^{\infty} t^{r-1} \exp \left(-i(2 v-E) t / \gamma^{1 / 2}\right) \exp \left(-t^{2} / 2\right) d t, \\
I_{r}^{-}(\gamma, E)=\frac{(-i)^{r} \gamma^{-r / 2}}{(r-1) !} \int_{0}^{\infty} t^{r-1} \exp \left(+i(2 v-E) t / \gamma^{1 / 2}\right) \exp \left(-t^{2} / 2\right) d t .
\end{gathered}
$$


Now from (A.1) and (A.2) it is obvious that $I_{r}^{+}$and $I_{r}^{-}$can be analytically continued to the whole $E$ - and $\gamma$-plane with a cut along the negative real $\gamma$-axis.

Next we want to derive bounds and an asymptotic expansion for $I_{r}^{+}$an $I_{r}^{-}$. To do this we first have to rewrite (A.1) and (A.2) in a slightly more general form: For $\beta \in(-\pi / 4+\varepsilon, \pi / 4-\varepsilon)$, define

$$
I_{r, \beta}^{ \pm}(\gamma, E)=\frac{( \pm i)^{r} \gamma^{-r / 2}}{(r-1) !} \int_{0}^{\infty} e^{i \beta} t^{r-1} \exp \left(\mp i(2 v-E) t / \gamma^{1 / 2}\right) \exp \left(-t^{2} / 2\right) d t
$$

Again by Cauchy's theorem we have for all $|\beta|<\pi / 4$,

$$
I_{r, \beta}^{ \pm}(\gamma, E)=I_{r}^{ \pm}(\gamma, E)
$$

In order to make (A.3) more suitable for expanding we make a variable substitution which yields:

$$
\begin{aligned}
I_{r, \beta}^{ \pm}(\gamma, E)= & \frac{( \pm i)^{r} \gamma^{-r / 2}}{2(r-1) !} e^{-i \beta r} \int_{0}^{\infty} t^{r / 2-1} \\
& \cdot \exp \left(\mp i e^{-i \beta}(2 v-E) \sqrt{t} / \gamma^{1 / 2}\right) \exp \left(-t e^{-2 i \beta} / 2\right) d t
\end{aligned}
$$

We shall now Taylor-expand the last factor in the integral and interchange sum and integral. In order to ensure convergence of the resulting integrals we have to restrict ourselves to

$$
\left|\arg \left(\mp i e^{-i \beta}(2 v-E) / \gamma^{1 / 2}\right)\right|<\pi / 2
$$

Since $\beta$ ranges from $-\pi / 4+\varepsilon$ to $\pi / 4-\varepsilon$ the range of validity of the asymptotic expansion will be

and

$$
-5 \pi / 2+2 \theta+\varepsilon<\arg \gamma<\pi / 2+2 \theta-\varepsilon \text { for } I_{r}^{+}
$$

$$
-\pi / 2+2 \theta+\varepsilon<\arg \gamma<5 \pi / 2+2 \theta-\varepsilon \text { for } I_{r}^{-} .
$$

Here $\theta=\arg (2 v-E)$.

By Taylor's theorem:

with

$$
\exp \left(-t e^{-2 i \beta} / 2\right)=\sum_{n=0}^{N} \frac{(-1)^{n}}{n ! 2^{n}} e^{-2 i \beta n} t^{n}+\tilde{R}_{N+1}(t),
$$

Inserting this into (A.4) yields:

$$
\left|\tilde{R}_{N+1}(t)\right| \leqq \frac{t^{N+1}}{(N+1) !}
$$

with $\quad I_{r, \beta}^{ \pm}(\gamma, E)=\frac{1}{(r-1) !}\left\{\sum_{n=0}^{N} \frac{\gamma^{n} \Gamma(2 n+r)}{n ! 2^{n}(2 v-E)^{r+2 n}}+R_{N+1, \beta}^{ \pm}(\gamma, E)\right\}$,

$$
\begin{aligned}
\left|R_{N+1, \beta}^{ \pm}(\gamma, E)\right| & \leqq \frac{|\gamma|^{-r / 2}}{2} \int_{0}^{\infty} t^{N+r / 2} \exp \left(-|2 v-E| t^{1 / 2} / C_{1}(\varepsilon)|\gamma|^{1 / 2}\right) d t \\
& =|\gamma|^{N+1} C_{1}(\varepsilon)^{r+2 N+2}|2 v-E|^{-(r+2 N+2)} 2^{-(N+1)} \frac{(2 N+r+1) !}{(N+1) !}
\end{aligned}
$$

where $C_{1}(\varepsilon) \rightarrow \infty$ for $\varepsilon \rightarrow 0$. 
We use the duplication formula for the $\Gamma$-function (see [11, p. 35]) to bring (A.9) and (A.10) into a slightly different form (henceforth we shall drop the subscript $\beta$ ), $I_{r}^{ \pm}(\gamma, E)=\pi^{-1 / 2}$

with

$$
(2 v-E)^{-r}\left\{\sum_{n=0}^{N}\left(\begin{array}{c}
2 n+r-1 \\
r-1
\end{array}\right)\left(\frac{2 \gamma}{(2 v-E)^{2}}\right)^{n} \Gamma(n+1 / 2)+R_{N+1}^{ \pm}(\gamma, E)\right\},
$$

$$
\left|R_{N+1}^{ \pm}(\gamma, E)\right| \leqq\left(\begin{array}{c}
2 N+r+1 \\
r-1
\end{array}\right) \Gamma(N+3 / 2)(2|\gamma|)^{N+1}\left(\frac{C_{1}(\varepsilon)}{|2 v-E|}\right)^{r+2 N+2} \text {. }
$$

Thus the estimate (A.12) shows the asymptoticity of the expansion (A.11) for $\gamma \rightarrow 0$ and a factorial bound for the remainder $R_{N+1}^{ \pm}(\gamma, E)$.

Finally we derive a bound for $I_{r}^{ \pm}$. Taking the expansion (A.11) up to zeroth order and estimating the remainder via (A.12) gives:

$$
\left|I_{r}^{ \pm}(\gamma, E)\right| \leqq\left\{1+\frac{r(r+1)}{2} \frac{|\gamma| C_{1}(\varepsilon)^{r+2}}{|2 v-E|^{2}}\right\} /|2 v-E|^{r} \leqq \frac{C_{2}(\varepsilon, \gamma)^{r}}{|2 v-E|^{r}}
$$

for some constant $C_{2}(\varepsilon, \gamma)$ with $C_{2} \rightarrow \infty$ for $\varepsilon \rightarrow 0$.

Acknowledgement. We are greatly indebted to Prof. Tom Spencer for giving us valuable hints for shortening the proofs.

\section{References}

1. Auberson, G., Mennessier, G.: Some properties of Borel summable functions. J. Math. Phys. 22, 2472-2481 (1981)

2. Constantinescu, F., Fröhlich, J., Spencer, T.: Analyticity of the density of states and replica method for random Schrödinger operators. J. Stat. Phys. 34, 571-596 (1984)

3. Constantinescu, F., Klöckner, K.: Absence of phase transitions and analyticity in the physical parameters. In: Studies in probability and related topics. Papers in honour of Octav Onicescu on his $90^{\text {th }}$ birthday. pp. 101-123. Demetrescu, M.C., Iosifescu, M. (eds.). Milano: Nagard 1983

4. Feller, W.: An introduction to probability theory and its applications. New York: Wiley 1966

5. Fröhlich, J., Mardin, A., Rivasseau, V.: Borel summability of the $1 / N$ expansion for the $N$-vector $[O(N)$ non-linear $\sigma]$ models. Commun. Math. Phys. 86, 87-110 (1982)

6. Glimm, J., Jaffe, A.: Quantum physics. A functional integral point of view. Berlin, Heidelberg, New York: Springer 1981

7. Hardy, G.H.: Divergent series. Oxford: Oxford University Press 1949

8. Jaffe, A.: Divergence of perturbation theory for bosons. Commun. Math. Phys. 1, 127-149 (1965)

9. Kunz, H., Souillard, B.: Sur le spectre des operateurs aux differences finies aleatoires. Commun. Math. Phys. 78, 201-246 (1980)

10. Nevanlinna, F.: Zur Theorie der asymptotischen Potenzreihen. Ann. Acad. Sci. Fenn. Ser. A 12, (1918-19)

11. Olver, F.W.J.: Asymptotics and special functions. New York, San Francisco, London: Academic Press 1974

12. Sokal, A.D.: An improvement of Watson's theorem on Borel summability. J. Math. Phys. 21, 261-263 (1980)

13. Wegner, F.: Exact density of states for lowest Landau level in white noise potential. Superfield representation for interacting systems. Z. Phys. B 51, 279-285 (1983)

Communicated by T. Spencer

Received February 27, 1984; in revised form October 15, 1984 
